\title{
Propuesta de Extensión de UML para Proceso de Conceptualización De Requisitos
}

\author{
Natalia Romero \\ Grupo de Investigación en Sistemas de Información \\ Departamento de Desarrollo Productivo y Tecnológico. Universidad Nacional de Lanús \\ Remedios de Escalada, Argentina \\ nataliab_romero@yahoo.com.ar
}

\begin{abstract}
El Proceso de Conceptualización de Requisitos, estructurado en una primera fase de Análisis Orientado al Problema y una segunda fase de Análisis Orientado al Producto, tiene como objetivo principal mejorar la comprensión y captura de los requisitos actuando como vinculo entre las actividades de educción de requisitos y modelado conceptual. No obstante, para mejorar aún más la calidad de los modelos que se obtienen como resultado de la actividad de conceptualización y facilitar la comunicación entre los distintos partícipes del proceso de software se propone en este trabajo una extensión de UML para dar soporte al proceso de conceptualización de requisitos con herramientas estándar y de uso difundido.
\end{abstract}

Index Terms-Conceptualización de requisitos, UML, Red de Petri, UML Extendido.

\section{INTRODUCCIÓN}

En este primer apartado se plantea el contexto del trabajo de investigación (sección A), se establece su objetivo (sección B), y se resume la estructura general del trabajo (sección C).

\section{A. Contexto del Trabajo de Investigación}

Un proceso de software es un conjunto de actividades y resultados que concluyen con un producto software específico [1]. Dentro de estas actividades se encuentra la de requisitos, tarea encargada de identificar, entender y especificar las necesidades del usuario [2]. Debido a su objetivo, esta actividad es de vital importancia dentro del ciclo de vida del software.

Dentro de la actividad de requisitos se encuentran dos subprocesos. En primer lugar, se encuentra la Educción de requisitos para adquirir el conocimiento y en segundo lugar está el Modelado Conceptual con el objetivo de construir modelos que describan la parte del mundo que se está analizando.

Sin embargo, se ha demostrado que existe una brecha conceptual en la transición de ambos subprocesos que demuestra una dificultad en la estructuración y categorización de la masa de información proveniente del proceso de educción que hace más compleja la comprensión del problema manifestado por el usuario [3][4].

A los efectos de evitar esta brecha conceptual, surge un modelo de procesos para la conceptualización de requisitos [5]. Este proceso tiene como principal actividad la conceptualización de requisitos con el objetivo de actuar como puente entre ambos subprocesos de la actividad de requisitos estableciendo una conexión adecuada entre los mismos y pudiendo "caracterizar" la información proveniente de la tarea de Educción de Requisitos para que luego puedan ser procesadas de manera más simple por la tarea de Modelado Conceptual.

Para poder "caracterizar" la información este proceso cuenta con determinadas tareas y técnicas que permitirán representar las necesidades del usuario. Para describir esta información el proceso de conceptualización de requisitos utiliza como elementos de representación los escenarios de usuario y el mapa de escenarios de usuario.

$\mathrm{Si}$ bien estos elementos permiten constituir una representación de la situación que se está analizando y permiten una mayor comprensión del problema manifestado por el usuario no utilizan herramientas de uso generalizado que podrían aportar una mayor calidad.

UML es una herramienta estándar que puede mejorar en gran medida la calidad del análisis y diseño obteniendo como resultado sistemas de mayor calidad. Al utilizar UML de manera interactiva, todos los partícipes del proceso de análisis $\mathrm{y}$ diseño de un sistema pueden conseguir una mayor comprensión de los requerimientos y los procesos que se deben realizar [6].

\section{B. Objetivo del Trabajo de Investigación}

Se ha propuesto como objetivo general del trabajo de investigación presentar una extensión de UML para dar soporte al proceso de conceptualización de requisitos. Esta extensión de UML buscará mejorar la calidad de los productos obtenidos de la conceptualización de requisitos permitiendo obtener modelos de mejor calidad y que faciliten aún más la comprensión de los requisitos a través del uso de herramientas estandarizadas.

Este trabajo de investigación definirá una extensión al lenguaje unificado de modelado que permita representar los objetivos del modelo de procesos de conceptualización de requisitos de manera completa.

Sin embargo, para poder realizar la derivación correspondiente del proceso de conceptualización de requisitos a modelos con base en extensiones de UML se deberá redefinir y adaptar determinadas tareas del proceso de conceptualización; aportando, además, las respectivas técnicas para poder llevar a cabo las nuevas tareas propuestas.

Por lo cual, el aporte de este trabajo consistirá en proponer una extensión de UML para el proceso de conceptualización de requisitos, readaptando el proceso en caso de ser necesario, para obtener modelos optimizados y de mayor calidad.

\section{Visión General del Trabajo de Investigación}

En la sección Introducción se plantea el contexto del Trabajo de Investigación, estableciendo en segundo lugar su 
principal objetivo y por último se resume la estructura del mismo.

En la sección Estado de la Cuestión se presentan distintos marcos teóricos que son de importancia para el objetivo del Trabajo de Investigación. Se presenta el marco teórico del modelo de proceso de Conceptualización de Requisitos, detallando su estructura y las tareas que lo conforman. Luego se introduce el marco teórico respectivo al Lenguaje Unificado de Modelado, definiendo conceptos orientados a objetos y describiendo las principales características y elementos componentes de UML. Para finalizar el apartado, se presenta la teoría referente a las Redes de Petri.

En la sección Descripción del Problema se identifica el problema de investigación demostrando los inconvenientes que podrían surgir de la utilización de formalismos de uso no difundido dentro del proceso de conceptualización de requisitos presentando la importancia de la aplicación de estándares dentro del proceso de desarrollo del software.

En la sección Solución se presenta una propuesta de extensión de UML con el uso del concepto de Redes de Petri para el proceso de conceptualización de requisitos. En este apartado se detallan el concepto de UML extendido y las modificaciones correspondientes de las tareas del proceso. Además, se introducen nuevas tareas con sus respectivas técnicas para poder derivar la conceptualización de requisitos en modelos de UML extendido.

En la sección Conclusiones se presentan los aportes de este trabajo y se destacan las futuras líneas de investigación que se consideran de interés en base al problema abierto que $\mathrm{se}^{\bullet}$ presenta en este trabajo de investigación.

En el Capítulo Referencias se listan todas las publicaciones consultadas para el desarrollo de este trabajo de Investigación.

\section{ESTADO DE LA CUESTIÓN}

En este apartado se presenta el estado de la cuestión sobre distintas teorías y técnicas que son concurrentes con los objetivos de este trabajo. Se presenta el marco teórico del Modelo de Proceso de Conceptualización de Requisitos (sección A), el marco teórico del Lenguaje Unificado de Modelado (sección B) y de Redes de Petri (sección C).

\section{A. Marco Teórico del Modelo de Proceso de Conceptualización de Requisitos}

En esta primera sección se realiza una definición del modelo de proceso para conceptualización de requisitos (1), luego se presenta su estructura (2) y se concluye con la presentación de las tareas que conforman al proceso y son de interés para este Trabajo de Investigación.

1) Definición del modelo de proceso de conceptualización de requisitos: Como primera medida es necesario definir el concepto de proceso. Un proceso es un conjunto de actividades relacionadas entre sí que se realizan sistémicamente con una finalidad específica [7]. Esa finalidad se lleva a cabo transformando elementos de entrada en resultados.

En segundo lugar, es importante aclarar el concepto de conceptualización de requisitos. La conceptualización de requisitos es una actividad que tiene como principal objetivo reducir la brecha conceptual entre el proceso de educción de requisitos de usuario al proceso de modelado conceptual [5]. Esta brecha conceptual puede observarse en la Fig. 1.

Con la introducción de la actividad de conceptualización de requisitos se espera, no sólo eliminar esa brecha conceptual como puede observarse en la Fig. 2, sino también eliminar la dificultad inherente a la comprensión de los problemas que presentan los usuarios y obtener modelos conceptuales de mayor calidad.

Este proceso de conceptualización de requisitos es el instrumento que permite llevar a cabo esta actividad.

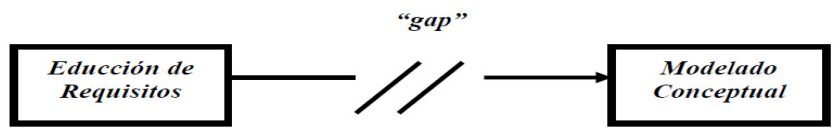

Fig. 1. Representación del "gap" entre los procesos de Educción de Requisitos y Modelado Conceptual [5]

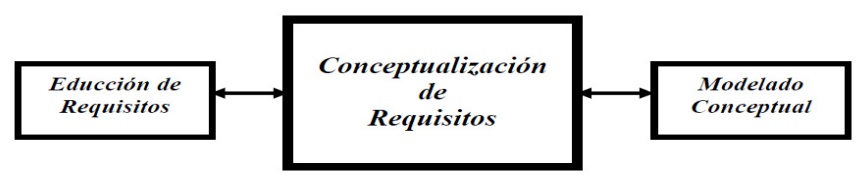

Fig. 2. Inserción de la actividad de "Conceptualización de Requisitos" entre las actividades de Educción de Requisitos y Modelado Conceptual [5]

2) Estructura del proceso de conceptualización de requisitos: La estructura que da soporte al proceso de conceptualización de requisitos está conformada por fases y tareas. Las dos fases están definidas por la terminología propuesta por Davis [3] dividiendo al proceso en dos:

La fase orientada al análisis del problema: tiene como finalidad conseguir una comprensión precisa del problema que el usuario espera resolver y presentar una posible solución al mismo.

La fase orientada al producto: especifica con exactitud las funcionalidades que el usuario espera obtener del software a desarrollar.

El proceso de conceptualización toma como elemento de entrada el Discurso del Usuario en Lenguaje Natural y obtiene como salida un conjunto de representaciones intermedias de los requisitos del usuario [5]

Como puede observarse en la Tabla 1, cada fase a su vez, está compuesta por determinadas tareas, que para ser llevadas a cabo necesitan de una técnica de transformación que les permita convertir los elementos de entrada en productos de salida.

La Figura 3 permite visualizar la relación entre cada una de las tareas a llevar a cabo y cuáles son las representaciones necesarias para cada una de ellas.

3) El Concepto de Escenario de Usuario: Un concepto que introduce este modelo, es el de Escenario de Usuario. Un Escenario de Usuario es definido como una descripción textual o gráfica de una situación determinada que tiene lugar en el ámbito de aplicación del producto software a desarrollar y que guarda una cierta relación con la realidad (y el problema a resolver) manifestada por el usuario en su discurso [5].

Estos escenarios de usuario, serán los que permitirán modelar tanto la descripción del problema (espacio problema) como las funcionalidades (espacio producto) que el usuario espera obtener del producto de software a desarrollar.

Un escenario de usuario está constituido fundamentalmente por los siguientes elementos:

- Actor: representa conceptos u objetos del mundo real relevantes para la situación analizada. 
TABLA I. REPRESENTACIÓN GRÁFICA DE LAS FASES, TAREAS Y PRODUCTOS CON SUS FORMATOS DE REPRESENTACIÓN [5]

\begin{tabular}{|c|c|c|c|c|c|c|}
\hline \multirow{2}{*}{ Fase } & \multirow{2}{*}{ Tarea } & \multicolumn{2}{|c|}{ Productos de Entrada } & \multirow{2}{*}{$\begin{array}{c}\text { Técnica de } \\
\text { Transformación a } \\
\text { Utilizar }\end{array}$} & \multicolumn{2}{|c|}{ Productos de Salida } \\
\hline & & Entrada & Representación & & Salida & Representación \\
\hline \multirow{3}{*}{$\begin{array}{l}\text { Análisis } \\
\text { Orientado al } \\
\text { Problema }\end{array}$} & $\begin{array}{l}\text { Segmentación del } \\
\text { Discurso de } \\
\text { Usuario }\end{array}$ & $\begin{array}{l}\text { Discurso de } \\
\text { Usuario }\end{array}$ & Texto Plano & $\begin{array}{l}\text { Técnica de Segmentación } \\
\text { de Discurso de Usuario }\end{array}$ & $\begin{array}{l}\text { Segmentos de } \\
\text { Texto }\end{array}$ & $\begin{array}{l}\text { Tablas de Segmentos } \\
\text { de Texto }\end{array}$ \\
\hline & $\begin{array}{l}\text { Análisis Cognitivo } \\
\text { de Segmentos de } \\
\text { Texto }\end{array}$ & $\begin{array}{l}\text { Segmentos de } \\
\text { Texto }\end{array}$ & $\begin{array}{l}\text { Tablas de Segmentos } \\
\text { de Texto }\end{array}$ & $\begin{array}{l}\text { Técnica de Identificación } \\
\text { de Conocimientos } \\
\text { Factuales, Procedurales, } \\
\text { Contextuales y de } \\
\text { Asociación }\end{array}$ & $\begin{array}{l}\text { Tipos de } \\
\text { Conocimiento }\end{array}$ & $\begin{array}{l}\text { Tabla de } \\
\text { Conocimientos } \\
\text { Factuales, } \\
\text { Procedurales y de } \\
\text { Asociación } \\
\end{array}$ \\
\hline & $\begin{array}{l}\text { Construcción del } \\
\text { Espacio Problema } \\
\text { en Escenarios de } \\
\text { Usuario }\end{array}$ & $\begin{array}{l}\text { Segmentos de } \\
\text { Texto } \\
\text { Tipos de } \\
\text { Conocimiento }\end{array}$ & $\begin{array}{l}\text { Tablas de Segmentos } \\
\text { de Texto } \\
\text { Tabla de } \\
\text { Conocimientos } \\
\text { Factuales, } \\
\text { Procedurales y de } \\
\text { Asociación }\end{array}$ & $\begin{array}{l}\text { Técnica de Construcción } \\
\text { del Diagrama Espacio } \\
\text { Problema de Escenarios de } \\
\text { Usuario }\end{array}$ & $\begin{array}{l}\text { Espacio } \\
\text { Problema de } \\
\text { Escenarios de } \\
\text { Usuario }\end{array}$ & $\begin{array}{l}\text { Diagrama Espacio } \\
\text { Problema de } \\
\text { Escenarios de Usuario }\end{array}$ \\
\hline \multirow{3}{*}{$\begin{array}{l}\text { Análisis } \\
\text { Orientado al } \\
\text { Producto }\end{array}$} & $\begin{array}{l}\text { Construcción de } \\
\text { Escenarios de } \\
\text { Usuario }\end{array}$ & $\begin{array}{l}\text { Segmentos de } \\
\text { Texto } \\
\text { Tipos de } \\
\text { Conocimiento de } \\
\text { Asociación } \\
\text { Espacio } \\
\text { Problema de } \\
\text { Escenarios de } \\
\text { Usuario } \\
\end{array}$ & $\begin{array}{l}\text { Tablas de Segmentos } \\
\text { de Texto } \\
\text { Tablas de Tipos de } \\
\text { Conocimiento de } \\
\text { Asociación } \\
\text { Diagrama de Espacio } \\
\text { Problema de } \\
\text { Escenarios de Usuario }\end{array}$ & $\begin{array}{l}\text { Técnica de Construcción } \\
\text { del Diagrama de } \\
\text { Escenarios de Usuario }\end{array}$ & $\begin{array}{l}\text { Escenarios de } \\
\text { Usuario }\end{array}$ & $\begin{array}{l}\text { Diagrama de } \\
\text { Escenarios de Usuario }\end{array}$ \\
\hline & $\begin{array}{l}\text { Refinamiento de } \\
\text { Escenarios de } \\
\text { Usuario }\end{array}$ & $\begin{array}{l}\text { Discurso de } \\
\text { Usuario } \\
\text { Escenarios de } \\
\text { Usuario } \\
\end{array}$ & $\begin{array}{l}\text { Texto Plano } \\
\text { Diagrama de } \\
\text { Escenarios de Usuario }\end{array}$ & $\begin{array}{l}\text { Técnica de Refinamiento } \\
\text { del Diagramas Escenarios } \\
\text { de Usuario }\end{array}$ & $\begin{array}{l}\text { Escenarios de } \\
\text { Usuario } \\
\text { Refinados }\end{array}$ & $\begin{array}{l}\text { Diagrama de } \\
\text { Escenarios de Usuario } \\
\text { Refinados }\end{array}$ \\
\hline & $\begin{array}{l}\text { Construcción de } \\
\text { Mapa Unificado de } \\
\text { Escenarios de } \\
\text { Usuario }\end{array}$ & $\begin{array}{l}\text { Segmentos de } \\
\text { Texto } \\
\text { Escenarios de } \\
\text { Usuario } \\
\text { Refinados } \\
\end{array}$ & $\begin{array}{l}\text { Tablas de Segmentos } \\
\text { de Texto } \\
\text { Diagrama de } \\
\text { Escenarios de Usuario } \\
\text { Refinados }\end{array}$ & $\begin{array}{l}\text { Técnica de Construcción de } \\
\text { Diagrama de Secuencia }\end{array}$ & $\begin{array}{l}\text { Mapa Unificado } \\
\text { de Escenarios de } \\
\text { Usuario }\end{array}$ & $\begin{array}{l}\text { Diagrama de Mapa } \\
\text { Unificado de } \\
\text { Escenarios de Usuario } \\
\text { Refinados }\end{array}$ \\
\hline
\end{tabular}

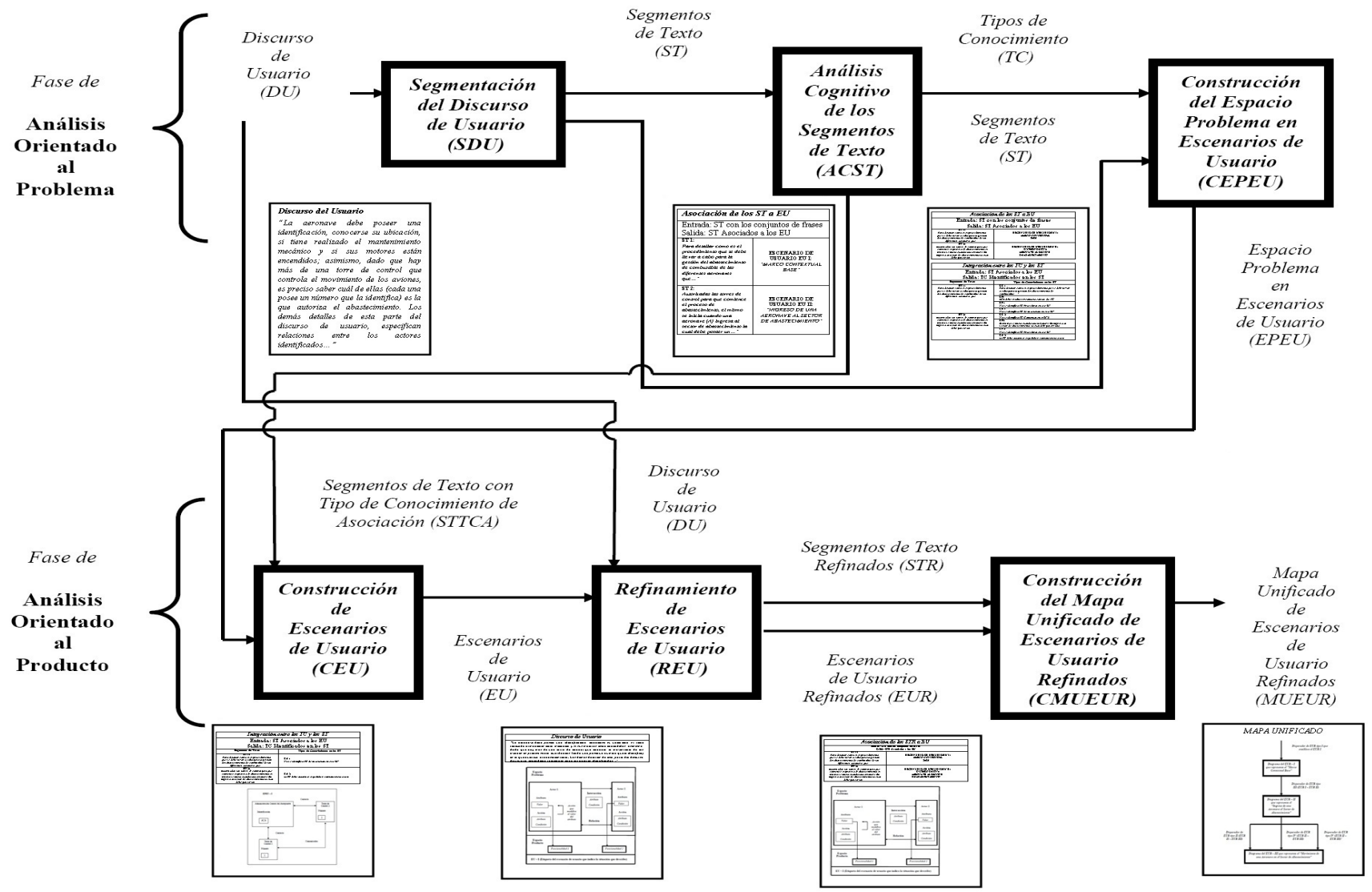

Fig. 3. Representación del proceso de Conceptualización de Requisitos 
- Atributos: propiedades que caracterizan a los actores y sus posibles estados.

- Relación: es una vinculación entre actores.

- Acción: determina el comportamiento de un actor.

- Interacción: determina como influye una determinada acción de un actor en el estado de otro.

- Funcionalidades requeridas por el usuario.

La figura 4 representa un escenario de usuario con los elementos mencionados anteriormente.

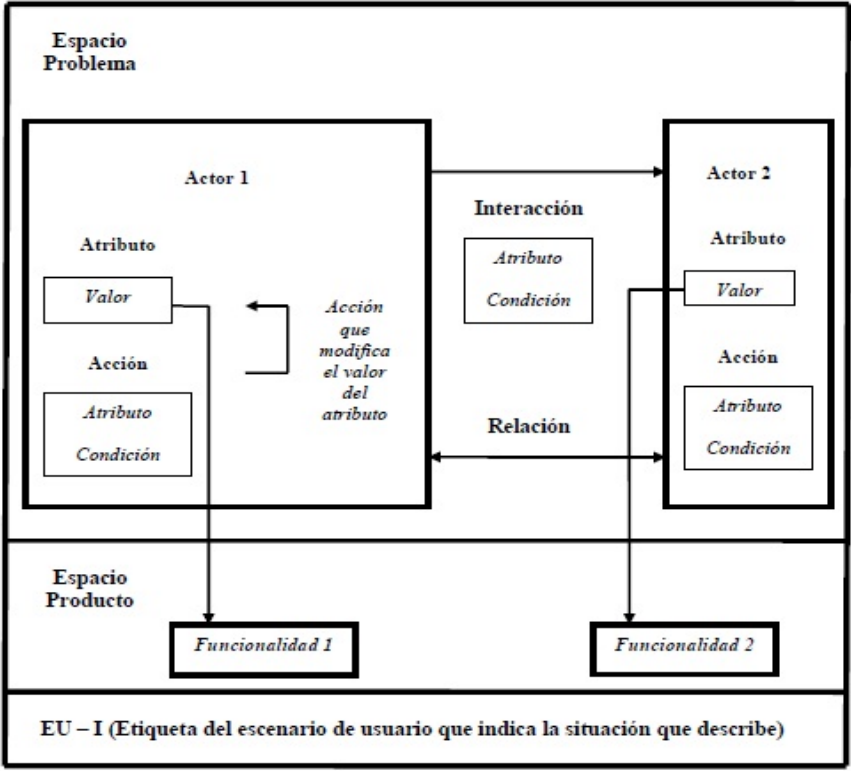

Fig. 4. Representación del concepto de Escenario de Usuario [5]

4) Tareas del proceso de conceptualización de requisitos: Como se mencionó en la sección anterior, cada una de las tareas necesita de un procedimiento para transformar los elementos de entrada, y dicho procedimiento está instrumentado con la utilización de técnicas de transformación. A continuación se presenta una breve descripción de cada una las tareas y técnicas que son el punto de partida para este Trabajo de Investigación.

a) Segmentación del Discurso de Usuario: La primera tarea a llevar a cabo es la Segmentación del Discurso de Usuario (DU) que tiene como finalidad analizar y dividir en frases cortas al discurso del usuario para luego integrarlas a determinados Segmentos de Texto (ST) y asociar los mismos a una situación específica presente dentro de la realidad analizada. Dicha situación se conoce con el nombre de Escenario de Usuario (EU).

b) Análisis Cognitivo de Segmentos de Texto: La segunda tarea a realizar dentro de la fase de análisis orientado al problema consiste en el Análisis Cognitivo de Segmentos de Texto. Aquí lo que se desea obtener son los distintos tipos de conocimiento que presenta cada uno de los segmentos de texto obtenidos de la tarea anterior. Para ello se debe procesar cada segmento de texto en busca de cuatro tipos de conocimiento:

- Contextual: relacionado con el ámbito o entorno de la situación.

- Factual: vinculado con frases declarativas, afirmaciones de hechos.

- Procedural: frases que indiquen procedimientos o secuencias de tareas.

- Asociación: funcionalidades y elementos involucrados para poder llevarla a cabo. c) Construcción del Diagrama de Espacio Problema de Escenarios de Usuario: La tercer y última tarea a realizar dentro de la fase de análisis orientado al problema consiste en la construcción de escenarios de usuario que representen el espacio problema, es decir una descripción de la situación que se está analizando y se espera resolver. Por medio de la técnica que permite la implementación de esta tarea, se hará uso de los tipos de conocimientos obtenidos en el paso anterior. Cada tipo de conocimiento permitirá detectar distintos elementos, a saber:

Contextual: identificar el ámbito de la situación analizada.

- Factual: identificar actores, atributos con sus respectivos valores, relaciones y posibles restricciones.

- Procedural: identificar acciones e interacciones que modifiquen los valores de los atributos de los actores.

Cada diagrama tendrá asociado un segmento de texto, que describa la situación que representa. Los elementos obtenidos a través del análisis de los tipos de conocimiento deben ser incorporados a los diagramas que correspondan.

El conocimiento de asociación será usado en la primera tarea de la fase de análisis orientado al producto ya que permitirá identificar las funcionalidades esperadas.

d) Construcción del Diagrama de Escenarios de Usuario: Es la primera tarea de la fase de análisis orientado al producto y como se destacó anteriormente, en esta tarea se hará uso del conocimiento de asociación. Este tipo de conocimiento permitirá detectar las funcionalidades que el usuario espera obtener del producto software y los actores que son necesarios para realizarlas.

Una vez identificadas las funcionalidades, se procede con la construcción del Diagrama de Escenario de Usuario Espacio Problema donde se incorporan dichas funcionalidades.

Por último, se vinculan los diagramas de Espacio Problema y Espacio Producto a través de los actores y las funcionalidades.

e) Refinamiento de Escenarios de Usuario: En esta tarea lo que se busca obtener es una revisión que permita detectar inconsistencias o ambigüedades. Esta revisión es llevada a cabo realizando un análisis que valide y depure el discurso de usuario original obteniendo como resultado un discurso de usuario refinado. A partir de allí se analizarán inconsistencias en los Segmentos de texto y Tipos de Conocimiento para luego validar y depurar los diagramas de escenarios de usuario.

f) Construcción del Mapa Unificado de Escenarios de Usuario Refinados: La última tarea de la fase de análisis orientado al producto tiene como finalidad analizar los escenarios de usuario para identificar elementos que permitan vincularlos y construir el mapa unificado que permitirá determinar la secuencia espacio temporal y las relaciones entre los escenarios de usuario.

\section{B. Marco Teórico del Lenguaje Unificado de Modelado (UML)}

En esta sección se presenta una introducción de conceptos del paradigma orientado a objetos (1), luego se describen generalidades de UML con sus principales características (2) detallando los principales componentes básicos de UML: elementos, relaciones y diagramas.

1) Conceptos Orientados a Objetos: El paradigma de la programación orientada a objetos (POO) surge con la 
necesidad de una respuesta al cambiante entorno de los negocios. Este enfoque es adecuado para situaciones en que los complicados sistemas de información requieren mantenimiento, adaptación y rediseño continuos. Un sistema orientado a objetos describe las entidades (cosas, lugares o personas relevantes para un sistema) como Objetos. Estos objetos a su vez se representan y agrupan en clases.

Cada clase a su vez define un conjunto de atributos y comportamientos que comparten cada objeto de esa clase. Los atributos describen propiedades que pueden tomar distintos valores dependiendo del objeto. Los comportamientos están representados por métodos que son las acciones que pueden realizar los objetos de una clase.

2) Generalidades de UML: UML es un lenguaje estándar que permite no solo modelar, sino también visualizar, especificar, construir y documentar desde sistemas de información empresariales hasta sistemas embebidos [8]. Para comprender mejor este concepto, se profundizará en la definición proporcionada:

- Es un lenguaje porque su principal función es comunicar. Proporciona un vocabulario y las reglas para combinar las palabras propias de ese vocabulario y hacer efectiva esa comunicación. A pesar de ser un lenguaje muy expresivo, es de fácil aprendizaje y utilización.

- Es un lenguaje estándar para modelado de software. Un modelo permite representar la realidad de forma simplificada. UML indica cómo crear y leer esos modelos.

- UML cubre la especificación y con esto se hace referencia a la construcción de modelos precisos, no ambiguos y completos.

- Los modelos UML pueden conectarse directamente con una amplia variedad de lenguajes de programación.

- UML cubre la documentación de los sistemas ya que proporciona un lenguaje para especificar desde los requisitos hasta el modelado de las actividades de planificación y gestión de versiones.

UML proporciona un conjunto estandarizado de herramientas para documentar el análisis y diseño de un sistema de software. Estas herramientas están divididas en bloques que incluyen: elementos, relaciones y diagramas.

Para comprender UML es fundamental entender sus tres bloques básicos de construcción y determinadas reglas que establecen como combinar los elementos de esos bloques básicos.

a) Elementos en UML: Un elemento puede definirse como una abstracción o un componente [6]. UML presenta cuatro tipos bien definidos de elementos:

- Estructurales: son las partes estáticas de un modelo, representan conceptos o cosas materiales. Dentro de este tipo se encuentran las clases, interfaces, casos de uso, componentes, etc.

- De comportamiento: son las partes dinámicas de un sistema. Son los verbos de un modelo, representan comportamiento en tiempo y espacio. Por ejemplo, máquinas de estado o una interacción.

- De agrupación: son las partes organizativas (partes en que puede descomponerse un modelo). Un ejemplo claro de este tipo de elemento son los paquetes que permiten incluir otros tipos de elementos para organizar el diseño de manera puramente conceptual.

- De anotación: son las partes explicativas, comentarios que describen, clasifican y hacen observaciones. Por ejemplo, las notas que permiten mostrar restricciones o comentarios.

b) Relaciones en UML: Otro de los bloques básicos de UML son las relaciones. Las relaciones tienen la función de vincular los distintos tipos de elementos. Dentro de esta categoría se pueden encontrar cuatro tipos de relaciones:

- Dependencia: es una relación semántica entre dos elementos donde un cambio en un elemento puede afectar directamente al otro elemento relacionado.

- Asociación: es una relación estructural que describe conexiones entre clases y objetos.

- Generalización: es una relación de especialización/generalización donde un elemento se basa en el otro elemento relacionado.

- Realización: es una relación semántica entre clasificadores.

c) Diagramas en UML: El último bloque básico de construcción de UML son los diagramas, una representación gráfica, que son los encargados de agrupar los distintos tipos de elementos con sus respectivas relaciones. Los diagramas permiten visualizar el sistema desde diferentes perspectivas:

- Diagramas Estructurales: describen la parte estática del sistema como ser un diagrama de clases o de componentes.

- Diagramas de Comportamiento: describen interacciones dentro del sistema, como ejemplo podría tomarse un diagrama de secuencias, de casos de uso o de actividades.

\section{Marco Teórico de las Redes de Petri}

Esta última sección introduce el concepto de una red de Petri tradicional (1) y luego se presenta el formalismo Diagrama de Desarrollo de Objetos Conceptuales inspirado en las redes de Petri (2).

1) Redes de Petri Clásicas: Las redes de Petri son una herramienta de modelado que fue inicialmente utilizada para el análisis de algoritmos en la computación paralela $\mathrm{o}$ concurrente [9], en la actualidad son un método de diseño de distintos tipos de sistemas y aplicaciones debido a su inherente permisividad y generalidad [10]. La importancia de su uso reside en la capacidad que presentan para visualizar el comportamiento dinámico de un sistema. Una red de Petri clásica se define como un grafo dirigido que posee:

- Lugares: representados por círculos tienen la función de relevar un estado en el que puede estar parte del sistema.

- Transiciones: representadas por barras rectangulares que permiten al sistema evolucionar por los distintos estados.

- Arcos dirigidos: que conectan las transiciones con los lugares y viceversa indicando el camino de evolución del sistema.

- Marcas: representan el estado del sistema en cada momento

2) Diagrama de Desarrollo de Objetos Conceptuales: Un diagrama de desarrollo de objetos conceptuales es un formalismo propuesto en base a las redes de petri para el 
modelado de las transformaciones de los objetos conceptuales. Este tipo de diagrama posee dos tipos de nodos. En primer lugar, se encuentran los objetos conceptuales denotados con círculos y las transformaciones que se representa con rectángulos [11].

\section{DESCRIPCIÓN DEL PROBLEMA}

En este apartado se presenta e identifica el problema de investigación a partir de las dificultades de obtener modelos resultantes del proceso de conceptualización de requisitos representados por formalismos de uso no difundido (sección A), se caracteriza el problema abierto (sección B) y se concluye con un sumario de investigación (sección $\mathrm{C}$ ).

\section{A. Identificación del Problema de Investigación}

La actividad de conceptualización de requisitos tiene el objetivo de facilitar la comprensión de los requisitos presentados por el usuario siendo una actividad puente entre las actividades de educción de requisitos y modelado conceptual [5].

El proceso de conceptualización de requisitos obtiene como resultado la representación gráfica de los requisitos de usuarios a través de un conjunto de Escenarios de Usuarios que permiten la comprensión del problema que se está analizando.

Sin embargo, los Escenarios de Usuario representan la información con formalismos de uso no extendido. Uno de los inconvenientes que podría ocasionar esta situación son los problemas de comunicación y entendimiento entre los distintos participantes de la producción de software.

Es importante mencionar que una derivación del proceso de conceptualización en representaciones con herramientas estándar permitiría un aumento de calidad y así también evitar problemas de entendimiento logrando un intercambio de información más precisa entre las distintas actividades del proceso de desarrollo de software.

En otras palabras, se podría concluir, que el modelo de proceso de conceptualización de requisitos, necesita ser adaptado de manera tal que permita obtener representaciones con formalismos estándares para evitar los inconvenientes mencionados anteriormente.

\section{B. Identificación del Problema de Investigación}

El problema abierto que se identifica en la presente sección, se caracteriza por la carencia de modelos representados con formalismos estándar en el proceso de conceptualización de requisitos.

Es de vital importancia en las distintas etapas de desarrollo de software usar estándares para obtener productos de mayor calidad, por lo cual para que el proceso de conceptualización de requisitos aumente la calidad de sus modelos es necesario realizar una derivación y adaptación del proceso para poder obtener diagramas que se representen con herramientas de uso difundido.

Las distintas herramientas estándares para modelar sistemas, como es el caso de UML, permiten, no sólo aumentar la calidad sino también mejorar la comunicación y la comprensión entre las distintas partes involucradas en la totalidad del proceso de desarrollo de software. Otra de las ventajas que aporta el uso de estos formalismos es obtener modelos no ambiguos que permitan ser interpretados de manera precisa evitando confusiones.

Sin embargo, la utilización solamente de UML podría no alcanzar para obtener modelos que representen completamente los resultados del proceso de conceptualización de requisitos.
Esta situación no genera nuevos inconvenientes, ya que UML es un lenguaje de modelado completamente flexible por lo cual podría ser adaptado y extendido con otros formalismos que permitan llevar a cabo la conceptualización de requisitos de manera efectiva.

Bajo este contexto, se pretende realizar un aporte con una propuesta de extensión de UML presentada en el apartado IV para dar soporte al Modelo de Proceso de Conceptualización de Requisitos.

\section{Sumario de Investigación}

De lo expuesto precedentemente surgen las siguientes preguntas de investigación:

Pregunta 1: ¿Se puede realizar una derivación del proceso de conceptualización de requisitos en modelos representados por UML Extendido?

Pregunta 2: ¿Cuáles son las extensiones que deben incluirse para obtener modelos que representen la totalidad del proceso de conceptualización de requisitos?

Pregunta 3: De ser posible la derivación del proceso de conceptualización de requisitos, ¿Cuáles son las nuevas tareas que deben ser incorporadas al proceso y cuáles deben ser modificadas para adaptarlas a un modelo de UML Extendido?

Se proponen soluciones a los interrogantes planteados en la próxima sección.

\section{SOLUCIÓN}

En esta parte se presenta: en primer lugar, una descripción general del modelo de conceptualización de requisitos (sección A), donde se detallan, en segundo lugar, la extensión de UML propuesta (sección B) describiendo el diagrama de UML extendido con Redes de Petri y la estructura general del modelo. Por último, se presenta una introducción a las técnicas incorporadas para la derivación posible de dicho modelo (sección C).

\section{A. Identificación del Problema de Investigación}

La construcción del modelo propuesto toma como base la estructura y el funcionamiento del modelo de proceso de conceptualización de requisitos desarrollado por Hossian [5]. A las tareas descriptas en dicho modelo se le han añadido nuevas actividades que permiten llevar a cabo una derivación del modelo en representaciones con elementos de UML extendidos utilizando Redes de Petri.

UML proporciona mecanismos estándar para poder realizar modelos que reflejen una representación real en este caso de los requisitos de un sistema.

UML permite construir y representar el vocabulario del problema (Análisis orientado al problema) y la solución (Análisis orientado al producto). Además, al proporcionar mecanismos de extensibilidad es posible incorporar el uso de herramientas adicionales, en este caso, las redes de Petri.

\section{B. Extensión de UML Propuesta}

En esta sección, se introduce el concepto de diagrama de UML extendido (parte A), describiendo en detalle los elementos que lo caracterizan y la propuesta de extensión en base a redes de Petri.

Además, se describen la estructura general del proceso (parte B) incluyendo fases, etapas y actividades con sus correspondientes salidas.

1) Diagrama de UML Extendido: El concepto de Diagrama de UML Extendido propuesto como solución de 
esta tesis para la conceptualización de requisitos está conformado por los formalismos tradicionales de UML y la adición de Redes de Petri. La caracterización de este concepto puede observarse en la Fig. 5.

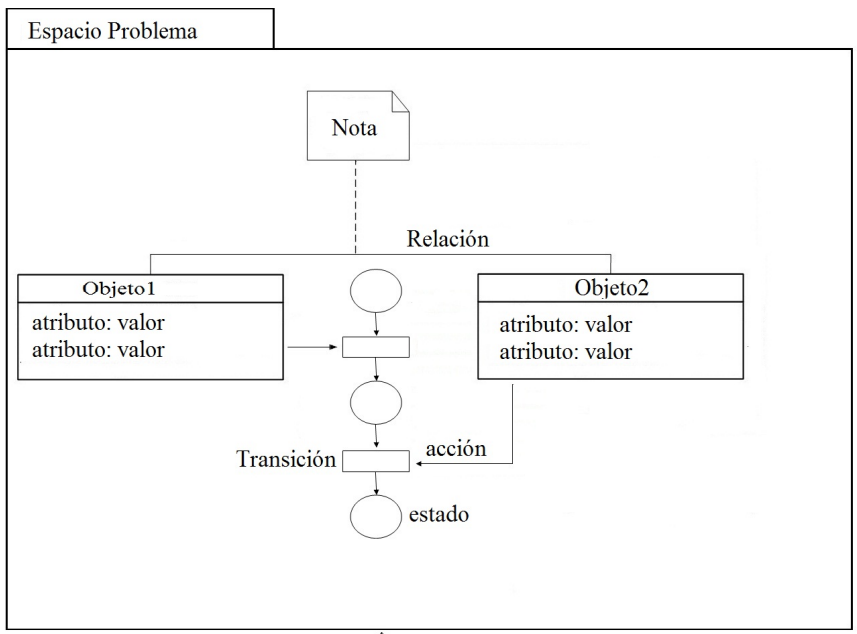

Espacio Producto

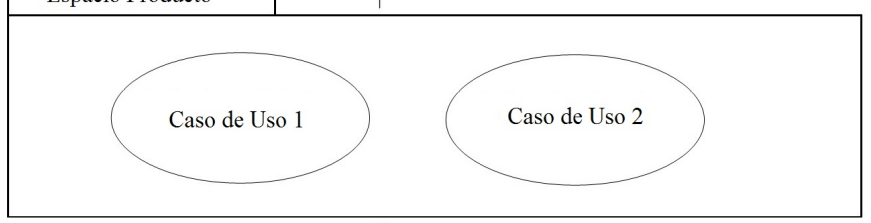

Fig. 5. Ejemplo de Diagrama de UML Extendido

UML será utilizado para poder representar y documentar los requisitos funcionales y la vista estática del sistema. En cuanto a las Redes de Petri se utilizarán para visualizar el sistema desde un punto de vista dinámico, reflejando el comportamiento entre los objetos a través de distintos estados y cambios generados por las distintas acciones que se realicen.

a) Elementos de UML: Se utilizarán distintos elementos de UML para representar el resultado del proceso de conceptualización de requisitos.

Dentro de los elementos de agrupación se utilizarán:

- Paquetes, para organizar el diseño. Permitirán diferenciar el espacio problema del espacio producto. Contendrán a los demás elementos UML.

De los elementos estructurales:

- Los objetos representarán la parte estática del sistema desde una perspectiva de instancias reales o prototípicas.

- Los casos de uso se utilizarán para indicar funcionalidades e irán acompañados por los escenarios de caso de uso para detallar información relativa a los mismos.

De los elementos de notación se usarán:

- Notas, permitirán mostrar restricciones y comentarios correspondientes.

Los elementos de comportamiento que caracterizan la parte dinámica del sistema serán representados a través de las Redes de Petri como se detalla en la sección siguiente.

UML también presenta cuatro tipos de relaciones que permitirán la vinculación entre los distintos elementos presentes en los diagramas. b) Extensión Propuesta: Las Redes de Petri son una herramienta que permite el modelado de la parte dinámica del sistema.

- Lugares para modelar los distintos estados de los objetos.

- Transiciones para representar las acciones de los objetos.

Además, es necesario destacar que, cómo se observa en la figura 6, las transiciones estarán conectadas al objeto que las accione.

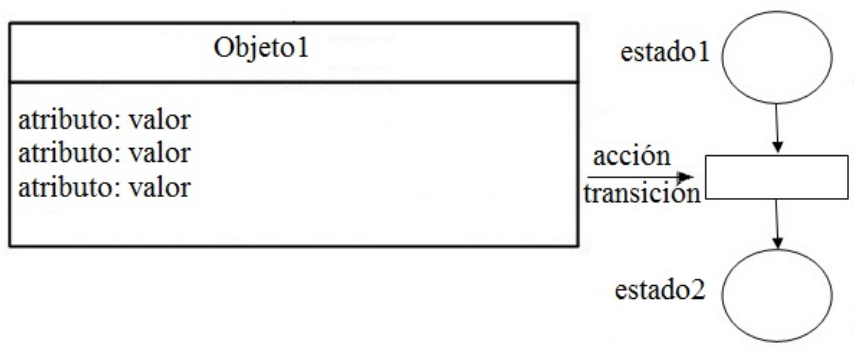

Fig. 6. Ejemplo de un Objeto conectado a una Red de Petri

Propuesta de Modelo de Proceso con UML Extendido para Conceptualización de Requisitos: Al modelo de proceso de conceptualización de requisitos desarrollado por Hossian [5] se han incorporado nuevas tareas y modificado las técnicas relativas a las actividades de construcción de los Diagramas para poder adaptarlas al concepto de UML Extendido.

Se mantiene la estructura de las dos fases principales (fase orientada al análisis del problema y fase orientada al análisis del producto) con la adición y modificación de algunas tareas dentro de cada fase. Puede observarse en la Tabla 2 (se encuentran resaltadas las modificaciones realizadas) una representación del proceso con sus correspondientes fases, tareas y técnicas a utilizar para poder realizar la conceptualización de requisitos con UML Extendido.

En cuanto a la fase de análisis orientada al problema se mantienen las tareas de Segmentación de Discurso de Usuario e Identificación de Tipos de Conocimiento con sus respectivas técnicas. La siguiente tarea a realizar será Identificación de Tipos de Elementos UML utilizando la Técnica de Identificación de Tipos de Elementos UML propuesta por esta tesis. En cuanto a la última tarea del modelo de proceso de conceptualización de requisitos [5], Construcción del Diagrama de Espacio Problema de Escenarios de Usuario, será reemplazada por la tarea Construcción del Diagrama UML Extendido de Espacio Problema utilizando la Técnica de Construcción del Diagrama UML Extendido de Espacio Problema.

Con respecto a la fase de análisis orientado al producto, la tarea de Construcción del Diagrama de Escenarios de Usuario será reemplazada por la incorporación de dos nuevas tareas:

- La tarea Identificación de casos de uso en los tipos de conocimiento con la utilización de una nueva técnica denominada Técnica de Identificación de casos de uso en los tipos de conocimiento.

- La tarea Construcción del Diagrama UML Extendido con la utilización de la Técnica Construcción del Diagrama UML Extendido correspondiente.

La modificación e incorporación de estas tareas en ambas fases permitirán al Ingeniero de Requisitos obtener una conceptualización de requisitos modelada con formalismos de UML extendidos con el uso de Redes de Petri. 


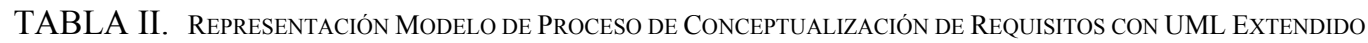

\begin{tabular}{|c|c|c|c|c|}
\hline \multicolumn{5}{|c|}{ MODELO DE PROCESO DE CONCEPTUALIZACIÓN DE REQUISITOS PARA UML EXTENDIDO } \\
\hline FASE & TAREA & TÉCNICA A UTILIZAR & ENTRADA & SALIDA \\
\hline \multirow{4}{*}{$\begin{array}{c}\text { ANÁLISIS } \\
\text { ORIENTADO } \\
\text { AL PROBLEMA }\end{array}$} & $\begin{array}{l}\text { Segmentación del Discurso de } \\
\text { Usuario }\end{array}$ & $\begin{array}{l}\text { Técnica de Segmentación de Discurso } \\
\text { de Usuario }\end{array}$ & Discurso de Usuario & Segmentos de Texto \\
\hline & $\begin{array}{l}\text { Análisis Cognitivo de } \\
\text { Segmentos de Texto }\end{array}$ & $\begin{array}{l}\text { Técnica de Identificación de } \\
\text { Conocimientos Factuales, } \\
\text { Procedurales, Contextuales y de } \\
\text { Asociación }\end{array}$ & Segmentos de Texto & Tipos de Conocimiento \\
\hline & $\begin{array}{l}\text { Identificación de Tipos de } \\
\text { Elementos UML }\end{array}$ & $\begin{array}{l}\text { Técnica de Identificación de Elementos } \\
\text { UML }\end{array}$ & $\begin{array}{l}\text { Tipos de Conocimiento } \\
\text { Segmentos de Texto }\end{array}$ & $\begin{array}{c}\text { Vinculación de Elementos } \\
\text { UML }\end{array}$ \\
\hline & $\begin{array}{l}\text { Construcción del Diagrama } \\
\text { UML Extendido Espacio } \\
\text { Problema }\end{array}$ & $\begin{array}{l}\text { Técnica de Construcción del Diagrama } \\
\text { UML Extendido Espacio Problema }\end{array}$ & $\begin{array}{l}\text { Vinculación de Elementos } \\
\text { UML }\end{array}$ & $\begin{array}{l}\text { Diagrama UML Extendido } \\
\text { Espacio Problema }\end{array}$ \\
\hline \multirow{4}{*}{$\begin{array}{c}\text { ANÁLISIS } \\
\text { ORIENTADO } \\
\text { AL PRODUCTO }\end{array}$} & $\begin{array}{l}\text { Identificación de Casos de Uso } \\
\text { en Conocimiento de } \\
\text { Asociación }\end{array}$ & $\begin{array}{l}\text { Técnica de Identificación de Casos de } \\
\text { Uso en Conocimiento de Asociación }\end{array}$ & $\begin{array}{l}\text { Tipo de Conocimiento de } \\
\text { Asociación }\end{array}$ & $\begin{array}{l}\text { Vinculación de Elementos } \\
\text { UML } \\
\text { Escenarios de Caso de Uso }\end{array}$ \\
\hline & $\begin{array}{l}\text { Construcción del Diagrama } \\
\text { UML Extendido }\end{array}$ & $\begin{array}{l}\text { Técnica de Construcción del Diagrama } \\
\text { UML Extendido }\end{array}$ & $\begin{array}{l}\text { Diagrama UML Extendido } \\
\text { Espacio Problema } \\
\text { Vinculación de Elementos } \\
\text { UML } \\
\text { Escenarios de Caso de Uso }\end{array}$ & $\begin{array}{l}\text { Diagrama UML Extendido } \\
\text { Completo }\end{array}$ \\
\hline & $\begin{array}{l}\text { Refinamiento de Diagramas } \\
\text { UML Extendidos }\end{array}$ & $\begin{array}{l}\text { Técnica de Refinamiento del } \\
\text { Diagramas UML Extendidos }\end{array}$ & $\begin{array}{c}\text { Discurso de Usuario } \\
\text { Diagramas UML Extendidos }\end{array}$ & $\begin{array}{c}\text { Diagrama UML } \\
\text { Extendidos Refinados }\end{array}$ \\
\hline & $\begin{array}{c}\text { Construcción de Diagrama de } \\
\text { Secuencia }\end{array}$ & $\begin{array}{l}\text { Técnica de Construcción de Diagrama } \\
\text { de Secuencia }\end{array}$ & $\begin{array}{c}\text { Segmentos de Texto } \\
\text { Refinados } \\
\text { Diagramas UML Extendido } \\
\text { Refinados }\end{array}$ & Diagrama de Secuencia \\
\hline
\end{tabular}

Por último, dentro de la fase de análisis orientado al producto se adaptará la tarea de Refinamiento de Escenarios de Usuario y se reemplazará la tarea de Construcción del Mapa Unificado [5] por las tareas de Refinamiento de Diagramas UML Extendidos y Construcción del Diagrama de Secuencias respectivamente.

La Figura 7 permite visualizar la relación entre cada una de las tareas a llevar a cabo y cuáles son las representaciones necesarias para cada una de ellas.

c) Identificación de Tipos de Elementos UML: Dentro de la Fase de Análisis Orientado al Problema se consideró necesario incorporar una tarea denominada Identificación de Tipos de Elementos UML. Esta tarea tiene como objetivo fundamental obtener, a partir de un análisis detallado de los tipos de conocimiento, los distintos elementos que caracterizan el modelado con UML.

Haciendo uso de la Técnica de Identificación de Tipos de Elementos UML, con la implementación de esta tarea se podrán obtener los componentes estructurales, de comportamiento y las relaciones para vincularlos, como así también se obtendrán las restricciones o comentarios que correspondan representados como elementos de notación.

d) Construcción del Diagrama UML Extendido Espacio Problema: Como resultado final de la fase de análisis orientado al problema se desea obtener un Diagrama UML Extendido que caracterice el problema principal de la situación analizada. Para obtener dicho Diagrama se debe implementar la tarea de Construcción del Diagrama UML Extendido Espacio Problema. De forma sintetizada, la utilización de esta técnica para llevar a cabo la tarea de construcción del Diagrama UML Extendido Problema permitirá incorporar y vincular todos los elementos detectados por la realización de la tarea de Identificación de Tipos de Elementos UML como así también desarrollar e incorporar la extensión basada en redes de Petri propuesta en esta tesis.

e) Identificación de Casos de Uso en Conocimiento de Asociación: La fase de análisis orientado al producto tiene como finalidad obtener las funcionalidades que se esperan obtener del producto a desarrollar. Por lo tanto, se propone una tarea de Identificación de Casos de Uso en Conocimiento de Asociación. Como es objetivo de esta tesis dar sustento al proceso de conceptualización de requisitos [5] con formalismos UML se propone esta tarea y su técnica correspondiente (Técnica de Identificación de Casos de Uso) para poder obtener a partir del análisis del tipo de conocimiento de asociación los casos de uso, siendo éstos los elementos estructurales de UML que permiten modelar las funcionalidades del sistema.

f) Construcción del Diagrama UML Extendido: La tarea de Construcción del Diagrama UML Extendido utiliza la Técnica de Construcción del Diagrama de UML Extendido. Esta tarea también pertenece a la fase de análisis orientado al producto y tiene como objetivo completar el Diagrama UML Extendido. 


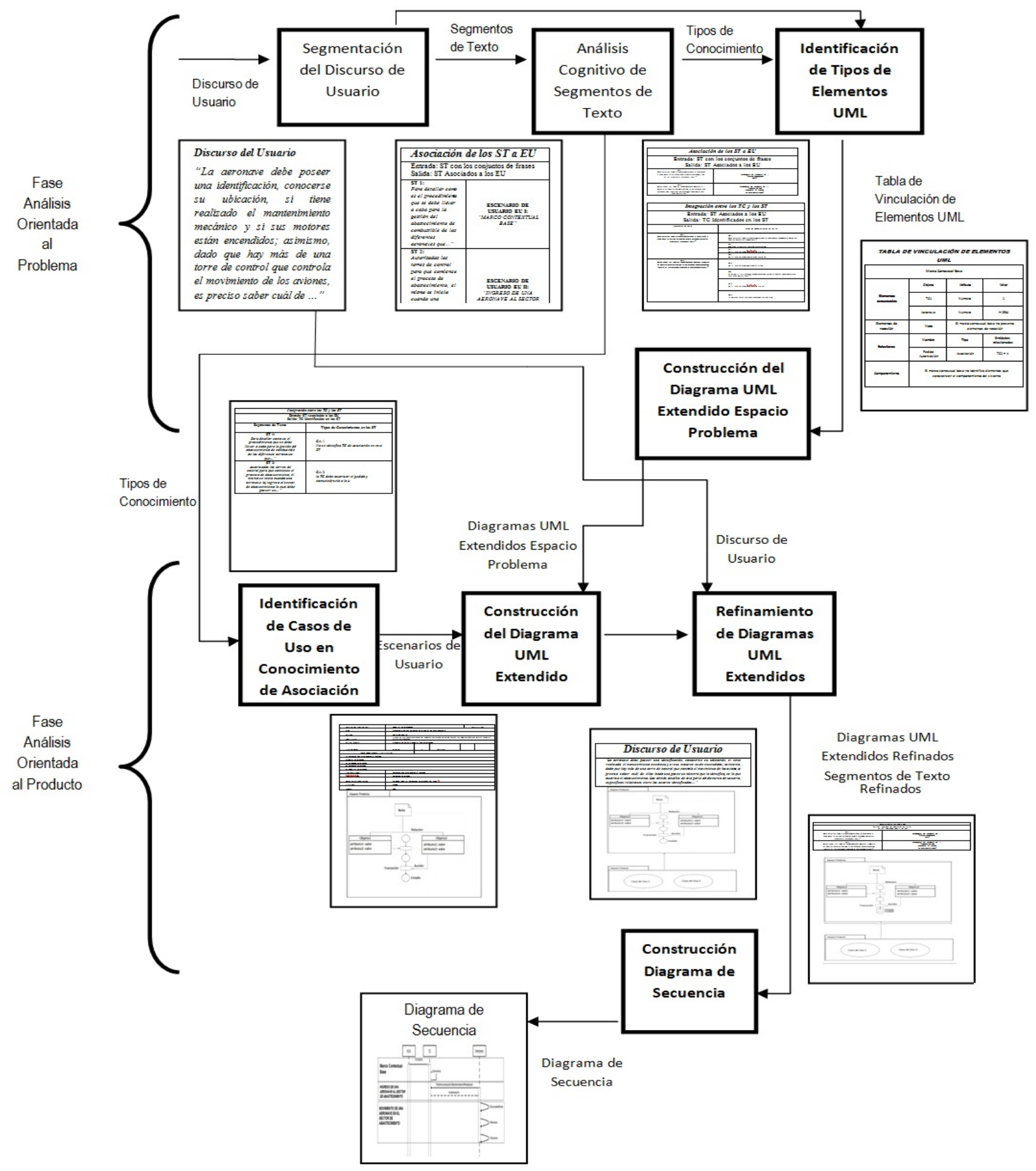

Fig. 7. Tareas propuestas para Conceptualización de Requisitos con UML Extendido

En otras palabras, se desarrollará la parte correspondiente al espacio producto que toma los casos de uso obtenidos en la tarea de Identificación de Casos de Uso y vincularlo al Diagrama UML Extendido Espacio Problema resultante de la realización de la última tarea de la fase de análisis orientado al problema: Construcción del Diagrama UML Extendido Espacio Problema

g) Refinamiento del Diagrama UML Extendido: Dentro de la fase de análisis orientado al producto también se encuentra la tarea denominada Refinamiento del Diagrama UML Extendido. La finalidad de esta tarea es contrastar el Discurso de Usuario con los diagramas obtenidos con el objetivo de determinar discrepancias entre ambos. Esta tarea utiliza la Técnica de Refinamiento de Diagramas UML Extendidos.

h) Construcción del Diagrama de Secuencias: La última tarea presente en la fase de análisis orientada al producto es la Construcción del Diagrama de Secuencias. Un diagrama de secuencias en UML tiene la función de representar sucesiones de interacciones entre clases u objetos y describir un escenario de caso de uso.

La implementación de esta tarea propone obtener, utilizando un diagrama de secuencias, una descripción final del orden de ejecución de los distintos diagramas UML Extendidos Obtenidos en la tarea de Refinamiento de Diagramas UML Extendidos. Puede observarse en la Fig. 8 un 
ejemplo de un Diagrama de Secuencia asociados los contextos que representan distintos Diagramas UML Extendidos.

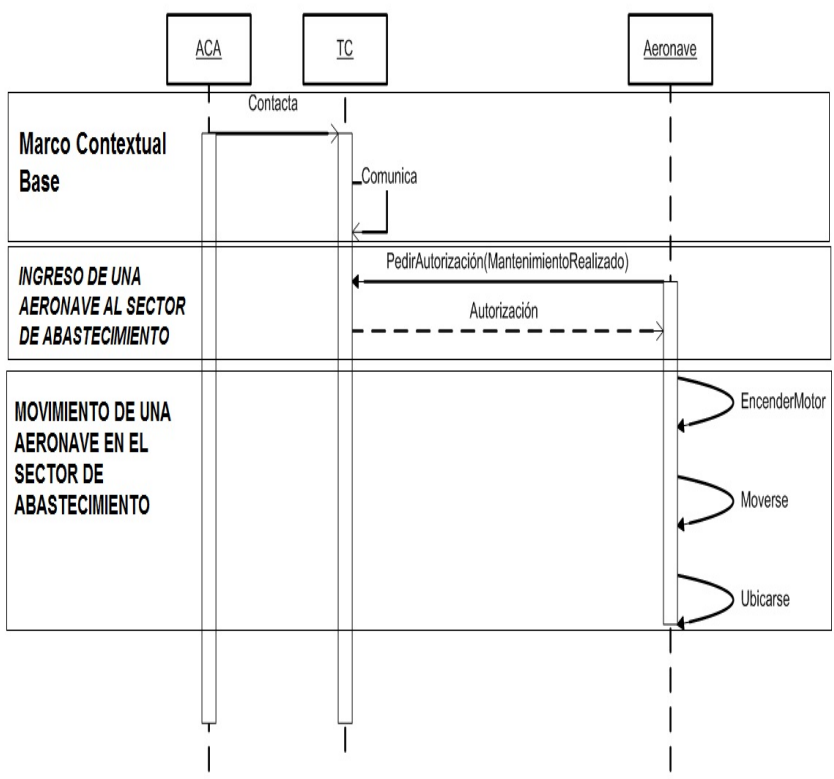

Fig. 8. Ejemplo de Diagrama de Secuencias

\section{Técnicas}

En esta sección se describen las nuevas técnicas que deben ser introducidas al proceso de conceptualización de requisitos para poder llevar a cabo las tareas propuestas en el apartado anterior. Se introducen las técnicas respectivas a la fase de análisis orientado al problema (1) y las técnicas de la fase de análisis orientado al producto (2).

1) Técnicas para la Fase de Análisis Orientado al Problema: Debido a la necesidad de incorporar nuevas tareas al modelo de proceso de conceptualización de requisitos [5] para poder darle sustento con formalismos UML y posibles extensiones, es indispensable la definición de nuevas técnicas para respaldar la realización de estas nuevas tareas.

Se ha incorporado una nueva técnica (a) y redefinido la técnica respectiva a la construcción del escenario de usuario (b) tanto para la fase de análisis orientado al problema como para la fase de análisis orientado al producto.

a) Técnica de Identificación de Elementos UML: Se incorpora al proceso de conceptualización de requisitos la Técnica de Identificación de Elementos UML que será utilizada por la tarea de Identificar Elementos UML. Está técnica tiene como finalidad analizar los tipos de conocimiento, que son producto de entrada, para detectar distintos tipos de elementos UML. Los pasos de aplicación de esta técnica, que pueden observarse en la Tabla 4.1, están basados en el uso de los tipos de conocimientos encontrados en los segmentos de texto y por último en la elaboración de una Tabla de Vinculación de Elementos (Tabla 3) que contendrá los distintos tipos de elementos UML encontrados.

El uso del tipo de conocimiento factual permitirá detectar:

- Elementos UML de tipo estructural, de los cuales se obtendrán los objetos a ser utilizados por el Diagrama de UML Extendido.

- Relaciones entre los objetos

- Elementos UML de anotación

El análisis del tipo de conocimiento procedural permitirá detectar los distintos estados y transiciones (acciones) que suceden entre los objetos para dar funcionamiento al sistema.
Este tipo de conocimiento detectará los elementos de UML de comportamiento, parte dinámica, que será modelada por las Redes de Petri.

TABLA III. TÉCNICA IDENTIFICACIÓN DE ELEMENTOS UML EN TIPOS DE CONOCIMIENTO

\begin{tabular}{|c|}
\hline $\begin{array}{l}\text { Téenica de identificación de elementos UML en los tipos de } \\
\text { conocimiento }\end{array}$ \\
\hline Entrada: Tabla segmentos de texto - tipo de conocimiento \\
\hline Salida: Tabla vinculación de tipos de elementos UML \\
\hline Paso 1: Asociar Tipos de conocimiento a Elementos UML \\
\hline Paso 2: Uso del conocimiento factual \\
\hline a. Identificar elementos estructurales \\
\hline b. Identificar relaciones \\
\hline c. Identificar elementos de anotación \\
\hline Paso 3: Uso del conocimiento procedural \\
\hline a. Identificar estados \\
\hline b. Identificar transiciones (acciones) \\
\hline Paso 4: Elaborar tablas de vin \\
\hline
\end{tabular}

El resultado final de la aplicación de esta técnica estará conformado por la Tabla de Vinculación de Elementos donde quedarán documentados todos los elementos UML detectados (Fig. 9).

\begin{tabular}{|c|c|c|c|c|c|}
\hline \multirow{5}{*}{$\begin{array}{l}\text { Elementos } \\
\text { estructurales }\end{array}$} & \multicolumn{2}{|c|}{ Objeto } & \multicolumn{2}{|c|}{ Atributo } & Valor \\
\hline & \multirow{2}{*}{\multicolumn{2}{|c|}{ Objeto1 }} & \multicolumn{2}{|c|}{ Atributo1 } & Valor1 \\
\hline & & & Atri & putoN & ValorN \\
\hline & \multirow{2}{*}{\multicolumn{2}{|c|}{ Objeto2 }} & Atri & buto1 & Valor1 \\
\hline & & & Atri & putoN & ValorN \\
\hline \multirow{2}{*}{$\begin{array}{l}\text { Elementos de } \\
\text { notación }\end{array}$} & \multirow{2}{*}{ Nota } & \multicolumn{4}{|c|}{ Nota1 } \\
\hline & & \multicolumn{4}{|c|}{ NotaN } \\
\hline \multirow{2}{*}{ Relaciones } & \multicolumn{3}{|c|}{ Tipo } & \multicolumn{2}{|c|}{$\begin{array}{l}\text { Entidades } \\
\text { relacionadas }\end{array}$} \\
\hline & \multicolumn{3}{|c|}{ Tipo de Relación } & \multicolumn{2}{|c|}{ Entidad1 - EntidadN } \\
\hline \multirow[t]{4}{*}{ Comportamiento } & Estado & \multicolumn{2}{|c|}{$\begin{array}{c}\text { Transición } \\
\text { anterior }\end{array}$} & \multicolumn{2}{|c|}{$\begin{array}{l}\text { Transición } \\
\text { Siguiente }\end{array}$} \\
\hline & Estado 1 & \multicolumn{2}{|c|}{ Transición 1} & \multicolumn{2}{|c|}{ Transición 2} \\
\hline & Estado 2 & \multicolumn{2}{|c|}{ Transición 2} & \multicolumn{2}{|c|}{ Transición 3} \\
\hline & Estado N & \multicolumn{2}{|c|}{ Transición 3} & \multicolumn{2}{|c|}{ Transición N } \\
\hline
\end{tabular}

Fig. 9. Representación de Tabla de Vinculación de Elementos

b) Construcción del Diagrama UML Extendido Espacio Problema: Además, como se mencionó anteriormente, para que pueda realizarse una correcta derivación del proceso de conceptualización de requisitos en diagramas de UML extendidos, será necesaria la modificación de la Técnica de Construcción del Diagrama de Espacio Problema utilizada en la Tarea de Construcción del Diagrama de Espacio Problema de Escenarios de Usuario. Se reemplazará su nombre por Técnica de Construcción de Diagramas de UML Extendidos de Espacio Problema (Tabla 4) y contendrá los pasos necesarios para la creación de los diagramas tomando como producto de entrada los segmentos de texto asociado a cada uno y la Tabla de vinculación de elementos UML.

Como primera medida deberá realizarse la construcción del diagrama correspondiente al Marco Conceptual Base. Dentro de este diagrama se reflejará sólo el contexto en el cuál se desarrolla el problema a resolver, Este diagrama no presenta aspectos de comportamiento de los objetos, por consiguiente, no será necesario la utilización de Redes de 
Petri. El primer paso para la construcción del Diagrama del Marco Contextual Base consiste en incorporar los objetos al paquete correspondiente al Espacio Problema del Marco Contextual Base. En segundo lugar, se incorporarán a los objetos los atributos y los valores pertenecientes a los mismos. Por último, se incorporan las relaciones permitiendo la vinculación de los objetos y obteniendo como resultado una imagen ilustrativa del contexto general del sistema. Tanto los objetos como los atributos, valores y relaciones son obtenidos de la Tabla de Vinculación de Elementos UML perteneciente al Marco Contextual Base.

TABLA IV. TÉCNICA CONSTRUCCIÓN DEL DIAGRAMA DE UML EXTENDIDO ESPACIO PROBLEMA

Técnica de Construcción del Diagrama de UML Extendido Espacio
Problema

Como segunda medida se realiza la construcción de los diagramas restantes. La forma de proceder es similar a la construcción del Diagrama Extendido del Marco Contextual Base con la adición de los pasos correspondientes para representar las acciones entre los objetos modelados a través de Redes de Petri. Por consiguiente, los pasos quedan resumidos de la siguiente manera:

- Incorporación de objetos al paquete correspondiente al Espacio Problema.

- Añadir los atributos y valores que definen a cada objeto.

- Incorporar las relaciones que permitan la interacción y comunicación entre los objetos.

- Para la elaboración de la Red de Petri primero se deben añadir los estados y las transiciones que los conectan. Luego se conectan las transiciones a los objetos para reflejar las distintas acciones que realizan modelando el comportamiento de los mismos.

- El último paso es añadir las Notas al paquete para poder modelar las restricciones o comentarios que se consideren esenciales.

2) Técnicas para la Fase de Análisis Orientado al Producto: Teniendo en cuenta la finalidad de la fase de análisis orientada al producto, cuyo objetivo fundamental es el reconocimiento de funcionalidades que se esperan obtener del producto de software a desarrollar, se propone la incorporación de una nueva técnica (a) al proceso de conceptualización requisitos desarrollado por Hossian [5], la redefinición de la Técnica de Construcción de Escenario de Usuario (b) como así también de la técnica de Refinamiento de Escenarios de Usuario convirtiéndola en Técnica de Refinamiento de Diagramas de UML Extendido (c) y la incorporación de la Técnica de Construcción de Diagramas de Secuencia (d).

a) Técnica de Identificación de Casos de Uso en Conocimiento de Asociación: La nueva técnica denominada Técnica de Identificación de Casos de Uso (Tabla 5) será utilizada en la tarea correspondiente a la Identificación de Casos de Uso en los Tipos de Conocimiento.

Para la aplicación de esta técnica serán necesarios el conocimiento de asociación y la tabla de vinculación de elementos UML correspondiente a cada segmento de texto como producto de entrada.

TABLA V. TÉCNICA DE IDENTIFICACIÓN DE CASOS DE USO EN CONOCIMIENTO DE ASOCIACIÓN

\begin{tabular}{|l|}
\hline Técnica de identificación de casos de uso en los tipos de conocimiento \\
\hline Entrada: Tabla segmentos de texto - tipo de conocimiento / Tabla \\
vinculación de tipos de elementos UML \\
Salida: Escenario de casos de uso \\
\hline Paso 1: Uso del conocimiento de asociación \\
a. Identificar de casos de uso \\
b. Identificar actores involucrados \\
c. Elaborar escenario de caso de uso \\
Paso 2: Vincular Elementos \\
\hline
\end{tabular}

La técnica está formada por dos pasos principales. El primero de ellos consiste en realizar uso y análisis del conocimiento de tipo de asociación para poder detectar las funcionalidades que se esperan obtener del producto software a desarrollar. Una vez obtenidas las mismas, que serán representadas por casos de uso, se deberán identificar los actores involucrados para realizarlas y por último elaborar los escenarios de casos de uso. Los escenarios de caso de uso son la descripción correspondiente a cada caso de uso y permitirán documentar principalmente la identificación, los pasos desempeñados y condiciones de cada uno de ellos (Fig. 10)

b) Técnica de Construcción del Diagrama UML Extendido: En cuanto a la Técnica de Construcción del Diagrama de Escenario de Usuario, utilizada en la tarea de Construcción del Diagrama de Escenario de Usuario, propuesta en el modelo de conceptualización de requisitos de Hossian [5] será reemplazada por la Técnica de Construcción del Diagrama UML Extendido (Tabla 6) para la tarea de construcción del mismo. Dicha técnica necesita como producto de entrada los Escenarios de Caso de Uso y los Diagramas de UML Extendidos resultantes de la Fase de Análisis Orientada al Problema.

La técnica consiste fundamentalmente en desarrollar el Diagrama correspondiente al Espacio Producto, compuesto por los casos de usos que le son producto de entrada, y vincular esos elementos al Diagrama de Espacio Problema correspondiente. Obteniendo como resultado el Diagrama UML Extendido Final. 


\begin{tabular}{|l|l|l|}
\hline $\begin{array}{l}\text { Nombre del caso } \\
\text { de uso: }\end{array}$ & Caso de Uso 1 & 0001 \\
\hline Área: & Área que representa el caso de uso \\
\hline Actores: & Actor 1 \\
\hline Descripción: & Descripción correspondiente al caso de uso \\
\hline Activar evento: & Acción que genere la activación del evento \\
\hline Tipo de señal & externa: & temporal: \\
\hline $\begin{array}{l}\text { Pasos desempeñados } \\
\text { principal) }\end{array}$ & \multicolumn{2}{|l|}{} \\
\hline Paso 1 & \multicolumn{2}{|l|}{} \\
\hline Paso N & Bajo qué condiciones puede hacerse \\
\hline Precondiciones & Que postcondiciones resultarán \\
\hline Poscondiciones & Puede tomar valores alta, media o baja \\
\hline Prioridad & Puede tomar valores alta, media o baja \\
\hline Riesgo &
\end{tabular}

Fig. 10. Representación de Escenario de Caso de Uso

TABLA VI. TÉCNICA DE CONSTRUCCIÓN DEL DiAgRAMA UML EXTENDIDO

\section{Técnica de Construcción del Diagrama de UML Extendido}

Entradas: Escenarios de caso de uso y Diagramas de UML Extendidos Espacio Problema

Salidas: Diagramas UML Extendidos

Paso 1: Construcción de los Diagramas de UML correspondientes al Espacio Producto

1.1 Incorporar Casos de Uso al Paquete Espacio Producto

Paso 2: Vincular Diagrama de Espacio Problema con Diagrama de Espacio Producto

2.1 Vincular casos de uso actores de Diagrama Espacio Problema

2.2 Vincular paquete espacio problema y paquete espacio producto

c) Técnica de Refinamiento del Diagrama UML Extendido: Esta técnica mantiene la estructura principal propuesta en el modelo de conceptualización de requisitos [5] con la adición de un paso para la validación y depuración de elementos UML (Tabla 7). Este paso extra incorporado tiene como finalidad evaluar los cambios que han sufrido los tipos de conocimiento en el paso anterior e impactarlos en la Tabla de Elementos UML y en los escenarios de caso de uso realizando las modificaciones correspondientes.

d) Técnica de Construcción del Diagrama de Secuencias: Por medio de la implementación de esta técnica se podrá llevar a cabo la última tarea, Construcción del Diagrama de Secuencias, de la Fase de Análisis Orientado al Producto. Se dispone como producto de entrada los Segmentos de Texto Refinados y los Diagramas UML Extendidos Refinados obtenidos como resultado de la realización de la tarea de Refinamiento de Diagramas UML Extendidos. Como producto de salida se obtendrá un Diagrama de Secuencias que le permitirá al usuario visualizar el orden de ejecución de cada uno de los Diagramas como así la vinculación entre los elementos presentes en los mismos.

La representación de esta técnica puede observarse en la Tabla 8.

Como principal medida se deben identificar en los productos de entrada los Objetos y las Interacciones que los mismos realizan. Luego se procederá con la construcción del Diagrama de Secuencias añadiendo los elementos identificados en el primer paso, los Focos de control para identificar los períodos de acción de los objetos y por último se vincularán los Diagramas UML Extendidos a las distintas interacciones presentes en el Diagrama de Secuencia.
TABLA VII. TÉCNICA DE REFINAMIENTO DEL DIAGRAMA UML EXTENDIDO

\begin{tabular}{|l|}
\hline Técnica de Refinamiento del Diagrama de UML Extendido \\
\hline Entradas: ST Asociados a los Diagramas y Diagramas UML Extendidos \\
Salidas: Diagramas UML Extendidos Refinados \\
\hline Paso 1. Análisis de Consistencia del Discurso de Usuario \\
1.1. Validación y Depuración de Incompletitudes \\
1.2. Validación y Depuración de Contradicciones \\
1.3. Validación y Depuración del Discurso de Usuario \\
Paso 2. Análisis de Consistencia de los Segmentos de Texto y Tipos de \\
Conocimiento \\
2.1. Validación y Depuración de los Segmentos de Texto \\
2.1.1. Incidencia del Discurso de Usuario Refinado en las "frases cortas" \\
2.1.2. Incidencia de las "frases cortas" en los Segmentos de Texto \\
2.2. Validación y Depuración de los Tipos de Conocimiento \\
2.2.1. Incidencia de los ST en la identificación del TC Contextual \\
2.2.2. Incidencia de los ST en la identificación del TC Factual \\
2.2.3. Incidencia de los ST en la identificación del TC Procedural \\
2.2.4. Incidencia de los ST en la identificación del TC de Asociación \\
Paso 3. Validación y Depuración de los Elementos UML \\
3.1. Incidencia de TC Factual en los elementos Estructurales \\
3.2 Incidencia de TC Procedural en los elementos de comportamiento \\
3.4 Incidencia de TC de Asociación en Escenarios de caso de uso \\
Paso 4. Validación y Depuración de los Diagramas UML Extendidos \\
4.1. Refinamiento de los Diagramas de UML Extendidos Espacio Problema \\
4.2. Refinamiento de los Diagramas de UML Extendidos Espacio Producto \\
4.3. Obtención de los Diagramas de UML Extendidos Refinados \\
Paso 4. Revisión Final de los Diagramas de UML Extendidos \\
Si los Diagramas de UML Extendidos son satisfactorios => Fin de la \\
Técnica \\
Si los Diagramas de UML Extendidos no son satisfactorios => Volver a \\
Paso I
\end{tabular}

TABLA VIII. TÉCNICA DE CONSTRUCCIÓN DEL DIAGRAMA DE SECUENCIAS

\section{Técnica de Construcción del Diagrama de Secuencias}

Entradas: Segmentos de Texto refinados y Diagramas UML Extendidos

Refinados

Salidas: Diagrama de Secuencias

Paso 1. Identificación de elementos

1.1. Identificar Objetos

1.2. Identificar interacciones entre los Objetos

Paso 2. Construir Diagrama de Secuencias

2.1 Incorporar Objetos y Línea de Vida

2.2 Incorporar Interacciones

2.3 Incorporar Foco de Control

2.4 Vincular interacciones a Diagramas UML Extendido

\section{CONCLUSIONES}

En este apartado se presentan las conclusiones obtenidas con el desarrollo de este trabajo de Investigación describiendo los aportes realizadas (sección A) Además, se mencionan futuras líneas de investigación a desarrollar a partir de este trabajo (sección B).

\section{A. Aportes del Trabajo de Investigación}

En este trabajo se ha presentado una propuesta de extensión de UML para dar soporte al proceso de conceptualización de requisitos. Se ha comprobado que realizando una adaptación de 
determinadas técnicas e incorporando nuevas que permitan llevar a cabo las tareas propuestas es posible obtener una descripción detallada del problema y de las funcionalidades que se esperan obtener representándolas a través de formalismos de uso generalizado como es el caso de UML. Sin embargo para obtener una representación completa, se propuso una extensión a dicho formalismo a través de la representación del comportamiento del sistema con el uso de Redes de Petri.

En este contexto, este trabajo ha propuesto las siguientes modificaciones al proceso de conceptualización de requisitos:

- Para la Fase de Análisis Orientado al Problema, se han propuesto dos nuevas tareas:

1. Identificación de tipos de elementos UML, está tarea toma como entrada los tipos de conocimientos y los segmentos de texto para obtener como producto de salida una Tabla de Vinculación de Elementos UML.

2. Construcción del Diagrama UML Extendido Espacio Problema, esta tarea necesita las Tablas de Vinculación de Elementos UML que serán representadas en los Diagramas UML Extendido Espacio Problema correspondientes.

Las técnicas que se incorporan para poder implementar estas tareas son la Técnica de Identificación de Elementos UML y la Técnica de Construcción del Diagrama UML Extendido Espacio Problema.

Como resultado final de esta fase se obtendrán los Diagramas UML Extendidos Espacio Problema que representarán una descripción de los elementos con sus respectivos comportamientos y restricciones del dominio del problema analizado.

- Para la Fase de Análisis Orientado al Producto, se han propuesto las siguientes tareas:

1. Identificación de los casos de uso: esta tarea necesita del tipo de conocimiento de asociación para obtener los escenarios de caso de uso que representen las funcionalidades que se esperan obtener.

2. Construcción del Diagrama UML Extendido: esta tarea tiene la finalidad de obtener los Diagramas UML Extendidos que representen tanto el espacio problema como el espacio producto, para ello se necesita como producto de entrada los Diagramas UML Extendidos Espacio Problema y los Escenarios de caso de uso.

3. Refinamiento de Diagramas UML Extendidos, esta tarea es una adaptación de la tarea de Refinamiento de Escenarios de Usuario propuesta en el modelo de proceso de conceptualización de requisitos [5]. Esta tarea permitirá obtener los Diagramas UML Extendidos refinados.

4. Construcción del Diagrama de Secuencias, esta tarea toma los Diagramas UML Extendidos finales para obtener una representación del orden de interacción de los distintos elementos de los diagramas.

Las técnicas necesarias para poder llevar a cabo estas tareas son la Técnica de Identificación de Casos de Uso, la Técnica de Construcción del Diagrama UML Extendido, la Técnica de Refinamiento de Diagramas UML Extendidos y la Técnica de Construcción de Diagrama de Secuencias.
Como resultado final de la realización de todas las tareas de la fase de análisis orientado al producto se podrá obtener una representación completa de la descripción del problema junto con las funcionalidades que se esperan obtener del sistema analizado.

Las tareas y técnicas que dan soporte a esta propuesta de Extensión de UML para el proceso de conceptualización de requisitos ha sido corroborada y validada en dos casos de validación de distintas características como ser el caso del Sistema de Abastecimiento de Combustible de Aeronaves en el Contexto de las Operaciones Aeroportuarias el caso del Sistema de Operaciones Bancarias por Cajero Automático.

Para concluir, con lo expuesto anteriormente quedan respondidas las preguntas planteadas en el apartado III sobre la posibilidad de poder realizar una derivación del proceso de conceptualización de requisitos con el objetivo de obtener modelos que utilicen formalismo de uso extendido como ser el caso de UML. En este caso, además, se ha propuesto una extensión a los formalismos UML con el uso de Redes de Petri para obtener modelos que representen la totalidad del proceso de conceptualización de requisitos.

\section{B. Futuras Líneas de Investigación}

Como consecuencia de este Trabajo de Investigación se plantean las siguientes líneas de trabajo surgidas durante el desarrollo del mismo:

Seguir analizando el problema y evaluar la posibilidad de incorporar nuevas extensiones a los formalismos de UML con la finalidad obtener representaciones aún más precisas que permitan reflejar las distintas situaciones que presenten los usuarios.

Para continuar evaluando la viabilidad de la extensión de UML propuesta para dar soporte al proceso de conceptualización de requisitos será necesario realizar experiencias de aplicación de la solución en una cantidad más amplia y variada de sistemas para la validación empírica.

\section{REFERENCIAS}

[1] Sommerville, I. Ingeniería de Software, Addison-Wesley. 2005.

[2] Kotonya, G., and Sommerville, I. Requirements Engineering: Processes and Techniques. John Wiley and Sons. 1998.

[3] Davis, A. 1993. Software Requirements: Objects, Functions and States; Prentice-Hall International.

[4] Faulk, S. Software Requirements: A Tutorial; In Software Engineering, IEEE Computer Society Press, 1997, pp 82-101.

[5] Hossian, A., "Modelo de Proceso de Conceptualización de Requisitos". Tesis Doctor en Ciencias Informáticas Universidad Nacional de La Plata. 2012.

[6] Kendall, K., Kendall, J., Análisis y Diseño de Sistemas. 6 ed. Pearson - Prentice - Hall, 2005.

[7] Curtis, B., Kellner, M., Over, J. 1992. Process Modelling. Communications of the ACM, 35(9): 75-90.

[8] Booch, G., Rumbaugh, J., Jacobson, I., The Unified Modeling Language User Guide. Pearson, 2005.

[9] Murillo Soto, L., "Redes de Petri: Modelado e Implementación de Algoritmos para Autómatas Programables". Tecnología en Marcha, Vol. 21, N 4, pp. 102-125, 2008.

[10] Tadao, M., "Petri Nets: Properties, Analysis and Applications". Proceedings of the IEEE, Vol. 77, $\mathrm{N}^{\circ} 4$, pp. 541-580, 1989.

[11] Rodríguez, D. y García-Martínez, R. "Propuesta de Proceso de Diseño de Espacios Virtuales de Trabajo Educativo Personalizables". Proceedings VIII Congreso de Tecnología en Educación y Educación en Tecnología, ISBN 978-987-167604-0. 
Natalia Romero. Licenciada en Sistemas por la Universidad Nacional de Lanús (UNLa). Su investigación se centra en "Extensiones de UML para el área de Conceptualización de Requisitos de Software". Fue Asistente de Investigación en el Laboratorio de Investigación y Desarrollo en Ingeniería del Software del Grupo de Investigación en Sistemas de Información (UNLa GISI) del Departamento de Desarrollo Productivo y Tecnológico de la Universidad Nacional de Lanús. 